\title{
REFRIGERATOR OPTIMAL SCHEDULING TO MINIMISE THE COST OF OPERATION
}

\author{
ROLAND BÁLINT AND ATtILA MAgyAR \\ Faculty of Information Technology, University of Pannonia, Egyetem u. 10, Veszprém, 8200, \\ HUNGARY
}

\begin{abstract}
The cost optimal scheduling of a household refrigerator is presented in this work. The fundamental approach is the model predictive control methodology applied to the piecewise affine model of the refrigerator.

The optimisation could not be solved using off-the-shelf tools, e.g. Multi-Parametric Toolbox, so a binary treebased optimal scheduling algorithm has been developed for this problem.
\end{abstract}

Keywords: optimal scheduling, model predictive control, operating cost minimization, modelling, day-ahead market

\section{Introduction}

Environmental awareness is trendy in modern societies. Policy-makers tend to prefer green energy, e.g. solar, wind, etc. in order to decrease carbon footprints. The demand-and-supply-based nature of the electricity market has led to hourly electricity prices in day-ahead markets [1]. Most people seek the cheapest solution by using their appliances during low price periods, however, there are certain devices, e.g. refrigerators work all day long. In this case, the only opportunity lies in the optimal scheduling of the cooling periods. The prescribed inside temperature of the refrigerator makes the problem difficult to solve.

\section{Problem Statement}

In a day-ahead market, the service provider provides the hourly electricity price for the next day. The problem to be solved is the optimal scheduling of a given refrigerator based on the forthcoming electricity prices. As a constraint, the refrigerator temperature must be kept within given boundary values.

\subsection{Energy Price}

The service provider provides the electricity price for the next 24 hours (DAM: day-ahead market). Fig.1 shows the electricity price [2] over a week, each line corresponds to the price of a day.

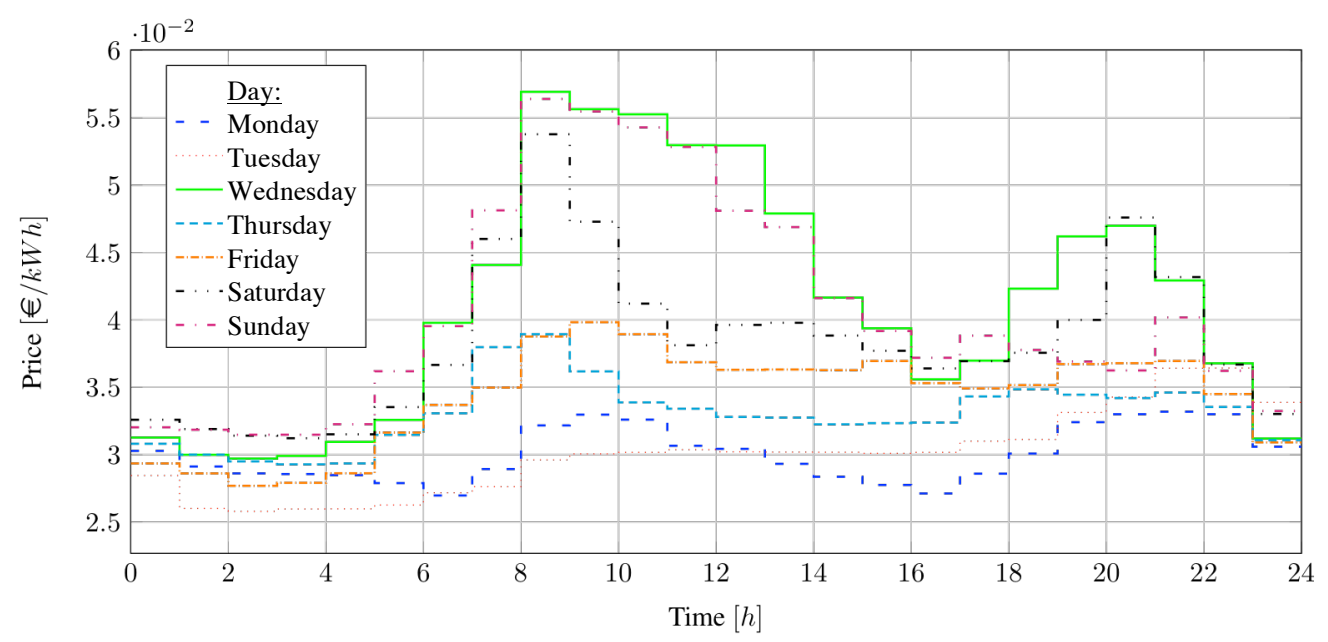

Figure 1. Electricity price of a day-ahead market over a week [2].

*Correspondence: balint.roland@virt.uni-pannon.hu 


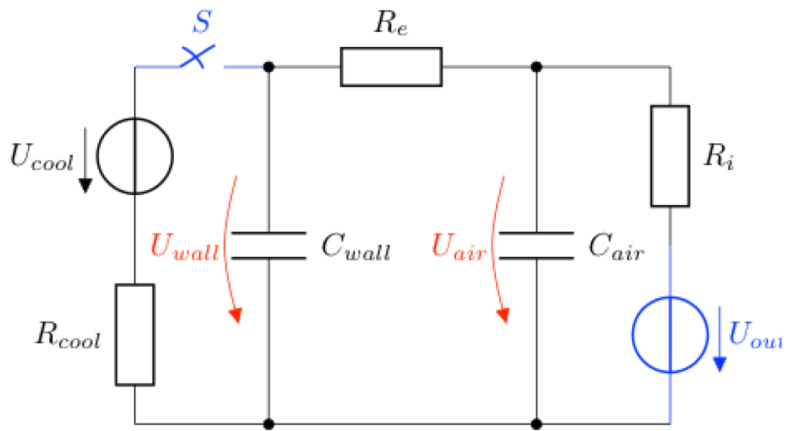

Figure 2. Electric substitution model of a refrigerator. $S$ symbolizes the switch of the devices controlled by a thermostat in an ordinary refrigerator.

\subsection{Refrigerator Model}

In order to describe the dynamics of the thermal behaviour of the refrigerator, the electric substitution model of Fig.2 can be used, where the red voltages correspond to the inner air temperature $\left(U_{\text {air }}\right)$ and the cooled back wall $\left(U_{\text {wall }}\right)$. The blue elements are the inputs to the system: the outer air temperature $\left(U_{\text {out }}\right)$ and the on/off switch $(S)$. The capacitors demonstrate the heat capacity of the back wall $\left(C_{\text {wall }}\right)$ and the inner air $\left(C_{\text {air }}\right)$ while the resistors are the heat transfer coefficients between the components.

\subsubsection{Cooling Dynamics}

The first case is when the switch $S$ is closed, i.e. the refrigerator is cooling. The dynamics of the system can be described by the state-space model of the form outlined in Eqs.(1) and (2), where the state and input vectors are given in Eqs.(3) and (4).

$$
\begin{gathered}
\dot{x}=\mathbf{A} \boldsymbol{x}+\mathbf{B} \boldsymbol{u} \\
y=\mathbf{C} \boldsymbol{x}+\mathbf{D} \boldsymbol{u} \\
\boldsymbol{x}=\left[\begin{array}{c}
T_{\text {air }} \\
T_{\text {wall }}
\end{array}\right] \\
\boldsymbol{u}=\left[\begin{array}{c}
T_{\text {out }} \\
S
\end{array}\right]
\end{gathered}
$$

The parameters of the state-space model of the cooling dynamics are given as follows:

$$
\begin{gathered}
\mathbf{A}_{\text {on }}=\left[\begin{array}{cc}
-\frac{R_{\mathrm{e}}+R_{\mathrm{i}}}{R_{\mathrm{e}} \cdot R_{\mathrm{R}} \cdot C_{\text {air }}} & \frac{1}{R_{\mathrm{e}} \cdot C_{\text {air }}} \\
\frac{1}{R_{\mathrm{e}} \cdot C_{\text {wall }}} & -\left(\frac{1}{R_{\mathrm{e}} \cdot C_{\text {wall }}}+\frac{1}{R_{\text {cool }} \cdot C_{\text {wall }}}\right)
\end{array}\right] \\
\mathbf{B}_{\text {on }}=\left[\begin{array}{cc}
\frac{1}{R_{\mathrm{i}} \cdot C_{\text {air }}} & 0 \\
0 & \frac{U_{\text {cool }}}{R_{\text {cool }} \cdot C_{\text {wall }}}
\end{array}\right] \\
\mathbf{C}=\left[\begin{array}{ll}
1 & 0 \\
0 & 1
\end{array}\right] \\
\mathbf{D}=\left[\begin{array}{ll}
0 & 0 \\
0 & 0
\end{array}\right]
\end{gathered}
$$

\subsubsection{Reheating Dynamics}

The second case is when the switch $S$ is open, i.e. the refrigerator is reheating to environmental temperature. The system is still linear and time invariant as outlined in Eqs.(3) and (4). The parameters of the state-space model are given in Eqs.(9)-(12).

$$
\begin{gathered}
\mathbf{A}_{\text {off }}=\left[\begin{array}{cc}
-\frac{R_{\mathrm{e}}+R_{\mathrm{i}}}{R_{\mathrm{e}} \cdot R_{\mathrm{i}} \cdot C_{\mathrm{air}}} & \frac{1}{R_{\mathrm{e}} \cdot C_{\mathrm{air}}} \\
\frac{1}{R_{\mathrm{e}} \cdot C_{\text {wall }}} & -\frac{1}{R_{\mathrm{e}} \cdot C_{\mathrm{wall}}}
\end{array}\right] \\
\mathbf{B}_{\text {off }}=\left[\begin{array}{cc}
\frac{1}{R_{\mathrm{i}} \cdot C_{\mathrm{air}}} & 0 \\
0 & 0
\end{array}\right] \\
\mathbf{C}=\left[\begin{array}{ll}
1 & 0 \\
0 & 1
\end{array}\right] \\
\mathbf{D}=\left[\begin{array}{ll}
0 & 0 \\
0 & 0
\end{array}\right]
\end{gathered}
$$

\subsection{Control Aim}

The aim of this study is to schedule refrigerator's operation to minimise the operational costs with the following assumptions:

- the operational cost is the energy consumption of the refrigerator during the day;

- the price of electricity changes hourly;

- the energy price is known for 24 hours in advance;

- the temperatures must be between the following operating constraints:

$\circ$ the inner air temperature should be between $0.1{ }^{\circ} \mathrm{C}$ and $5.5^{\circ} \mathrm{C}$;

o the cooled back wall temperature should be between $-19{ }^{\circ} \mathrm{C}$ and $7{ }^{\circ} \mathrm{C}$;

- the outer air temperature is constant;

- the input variable is binary $(O n / O f f)$;

- the models should be chosen based on the value of the input.

The main objective is to minimize the operational costs, defined as

$$
\text { cost }=\sum_{k=0}^{N-1}\left(p_{\mathrm{k}} \cdot U_{\mathrm{k}}\right)
$$

where $p_{\mathrm{k}}$ is the price of electricity and $U_{\mathrm{k}}$ is the input at time $T_{\mathrm{s}} \cdot k\left(T_{\mathrm{s}}\right.$ : sample time $)$.

\section{Optimal scheduling}

To find the optimal scheduling of a system the model predictive control approach is used. Afterwards a novel heuristic optimisation technique is presented.

\subsection{Model Predictive Control of the System}

For optimization, the Multi-Parametric Toolbox 3.0 (MPT) [3] is used first, because in this Matlab Toolbox we can find all the necessary options can be found:

- 'Piecewise Affine' (PWA) modelling for linear time invariant (LTI) systems;

- optimal controller design for PWA systems. 
The solver uses a cost function as follows:

$$
\min \sum_{k=0}^{N-1}\left(\left\|\boldsymbol{Q}_{\mathrm{x}} \boldsymbol{x}_{\mathrm{k}}\right\|_{\mathrm{p}}+\left\|\boldsymbol{Q}_{\mathrm{u}} \boldsymbol{u}_{\mathrm{k}}\right\|_{\mathrm{p}}\right)
$$

where variables $\boldsymbol{x}_{\mathrm{k}}$ and $\boldsymbol{u}_{\mathrm{k}}$ are the state and input vectors at the $k^{\text {th }}$ step of the prediction horizon $\mathrm{N}$. The $\|\cdot\|_{\mathrm{p}}$ expression is the standard p vector norm, and $\boldsymbol{Q}_{\mathrm{X}}$ and $\boldsymbol{Q}_{\mathrm{u}}$ are penalty matrices.

The MPT toolbox for the PWA system description uses discrete time models, thus initially the continuous time model must be discretised. However, there are two problems to solve:

- the penalty matrices $\left(\boldsymbol{Q}_{\mathrm{x}}\right.$ and $\left.\boldsymbol{Q}_{\mathrm{u}}\right)$ cannot be changed, so the actual electricity price cannot be used in this form;

- the time information of the system, e.g. system time, is unknown.

To handle these problems, two supplementary state variables have been introduced. The first counts the time and the second stores the actual energy price. If the refrigerator is switched off the energy price is zero, otherwise it provides the actual price value. One pair of $(\mathrm{On} / \mathrm{Off})$ systems for every price of each hour needs to be created, during the calculation the model parameters and the price are constants. Thus, the choice is based on the state of the binary input and the time value of the state vector. The modified LTI state-space models are the following:

$$
\begin{aligned}
& \boldsymbol{x}=\left[\begin{array}{c}
T_{\text {air }} \\
T_{\text {wall }} \\
t \\
p
\end{array}\right] \\
& \boldsymbol{u}=[S] \\
& \boldsymbol{\Phi}_{\text {on }}=\left[\begin{array}{cccc}
0.9998 & 0.0001 & 0 & 0 \\
0.0004 & 0.9977 & 0 & 0 \\
0 & 0 & 1 & 0 \\
0 & 0 & 0 & 0
\end{array}\right] \\
& \boldsymbol{\Gamma}_{\mathrm{on}}=\left[\begin{array}{c}
-0.0024 \\
-0.045 \\
0 \\
p_{\mathrm{h}}
\end{array}\right] \\
& \boldsymbol{\Phi}_{\text {off }}=\left[\begin{array}{cccc}
0.9998 & 0.0001 & 0 & 0 \\
0.0010 & 0.9988 & 0 & 0 \\
0 & 0 & 1 & 0 \\
0 & 0 & 0 & 0
\end{array}\right] \\
& \boldsymbol{\Gamma}_{\text {off }}=\left[\begin{array}{c}
0 \\
0 \\
0 \\
p_{\mathrm{h}}
\end{array}\right] \\
& \boldsymbol{f}=\left[\begin{array}{c}
0.0022 \\
0.028 \\
1 \\
0
\end{array}\right] \\
& \mathbf{C}=\left[\begin{array}{llll}
1 & 0 & 0 & 0 \\
0 & 1 & 0 & 0 \\
0 & 0 & 1 & 0 \\
0 & 0 & 0 & 1
\end{array}\right]
\end{aligned}
$$

Table 1. The simulation time in minutes versus horizon size.

$$
\begin{aligned}
& \begin{array}{c|cccc}
\text { Size of horizon, }- & 8 & 10 & 12 & 13 \\
\text { Calculation time, min } & 2 & 8 & 59 & 111
\end{array} \\
& \mathbf{D}=\left[\begin{array}{llll}
0 & 0 & 0 & 0 \\
0 & 0 & 0 & 0 \\
0 & 0 & 0 & 0 \\
0 & 0 & 0 & 0
\end{array}\right]
\end{aligned}
$$

The hourly changing energy prices are denoted by $p_{\mathrm{h}}$ in matrices $\boldsymbol{\Gamma}$, so there are 24 models with two system dynamics ( $O n$ and $O f f$ ) in total. The model actually used is chosen based on the input (On and Off states) and the third element of the state vector ( $t$ : define the model over time so the energy price value). Based on the two variables the necessary model is chosen:

- if the refrigerator operates, its value is the actual energy price;

- if the refrigerator is switched off, it is zero.

By the summation of the values of $x_{4}$ the operational cost is obtained (in $€ / \mathrm{kWhr}$ ) for the operating period. The piecewise affine model of the system is given by $E q .(24)$.

$$
\boldsymbol{x}_{\mathrm{k}+1}= \begin{cases}\boldsymbol{\Phi}_{\mathrm{on}} \boldsymbol{x}_{\mathrm{k}}+\boldsymbol{\Gamma}_{\mathrm{on}} \boldsymbol{u}_{\mathrm{k}}+\boldsymbol{f}, & \text { if }[\boldsymbol{S}]=1 \\ \boldsymbol{\Phi}_{\mathrm{off}} \boldsymbol{x}_{\mathrm{k}}+\boldsymbol{\Gamma}_{\mathrm{off}} \boldsymbol{u}_{\mathrm{k}}+\boldsymbol{f}, & \text { if }[\boldsymbol{S}]=0\end{cases}
$$

The simulation is very sensitive to some design parameters. These parameters are the sampling time $\left(T_{\mathrm{S}}\right)$, the prediction horizon size, and the cycle number of the simulation horizon. The correlation between horizon size and simulation time is outlined in Table 1 $\left(T_{\mathrm{S}}=5 \mathrm{~min}\right)$. If the horizon is bigger than $12-15$, the simulation will end in deadlock. The sample time value cannot be too large because the change in one step would be too high and the algorithm would not be able to function properly. The multiplication of the sample time and the horizon yields to predicted time horizon. If the sample time and the horizon are small, the algorithm runs quickly, but with a small pre-determined time interval and cannot calculate the forthcoming electricity prices. As $N_{\text {sim }}$ increases, the running accelerates. In the PWA system, only the systems of the simulated time interval are present. If $N_{\text {sim }}$ is small, the number of models is small so the algorithm must choose from fewer systems but in that case, the cycle has to run repeatedly as the new PWA system is defined.

\subsection{Heuristic Search Algorithm using Binary Tree Growing}

The general optimizer in the MPT is very slow due to several problem-specific assumptions, e.g. binary input, and the horizon size is bounded so another optimisation algorithm is needed that runs faster and uses problemspecific heuristics. 


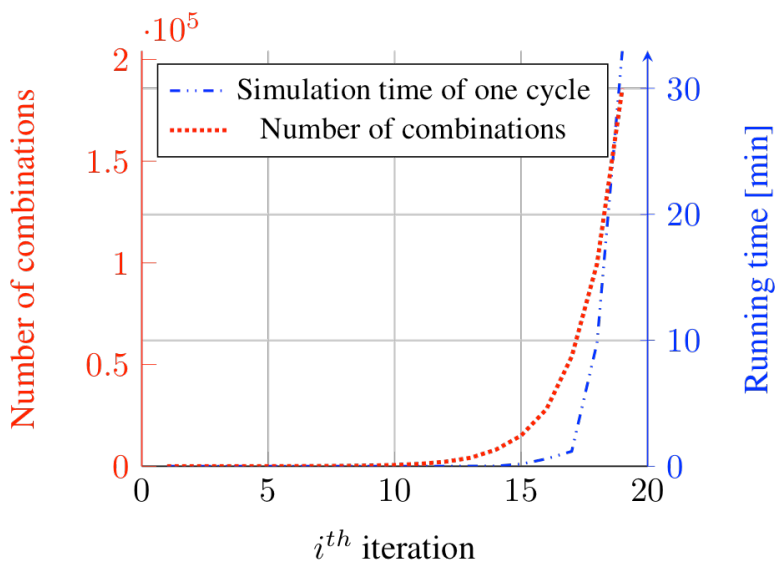

Figure 3. Running time and number of combinations vs number of cycles.

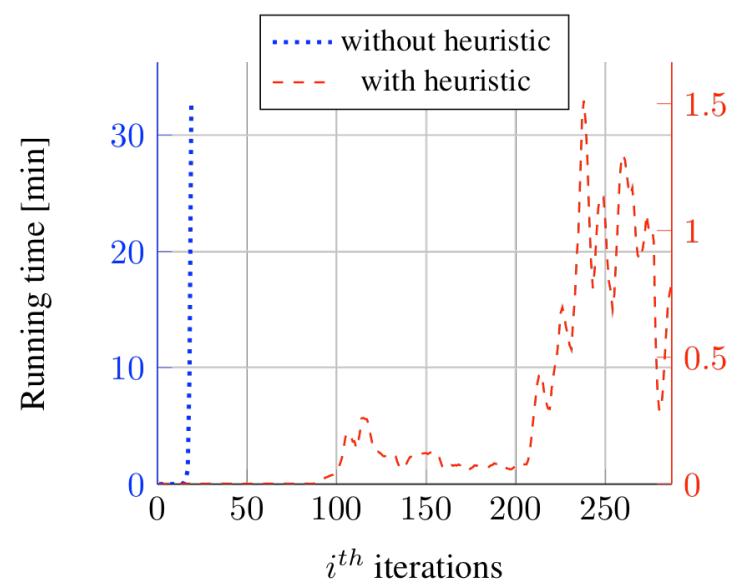

Figure 4. Without the heuristic algorithm, the simulation time of the $20^{\text {th }}$ cycle is more than 30 minutes, but with the heuristic algorithm, it has a maximum of 1.5 minutes.

\subsubsection{Base of Algorithm}

In order to find the optimal solution, all input combinations need to be counted over time. Trivially, this number can be reduced by deleting those combinations where the system exceeds the operating constraints. In this case, in all steps, the states are duplicated and 1 or 0 (On or $O f f$ ) is added to input vectors so the number of combinations grow exponentially and the simulation time of one cycle grows, too (Fig.3). Without additional heuristics, the running time of the $18^{\text {th }}$ loop was as high as the MPT simulation with a horizon size of 13 .

Model-related heuristics can be applied to decrease the number of combinations. This rule is simple: between any two input combinations, if the corresponding air and wall temperatures are higher and also the operational cost is greater than or equal to one of the examined combinations then this input combination is not optimal, i.e. it can be ignored. The pseudo code of the algorithm is summarized by Algorithm 1. With these heuristics, the number of combinations and the running time of the simulation can be reduced. Fig.4 shows the running time of the $n^{\text {th }}$ cycle with and without this filtering. This figure shows that without the filter, the simulation time of the $20^{\text {th }}$ cycle is more than 30 minutes but with the filter it is a maximum of 1.5 minutes.

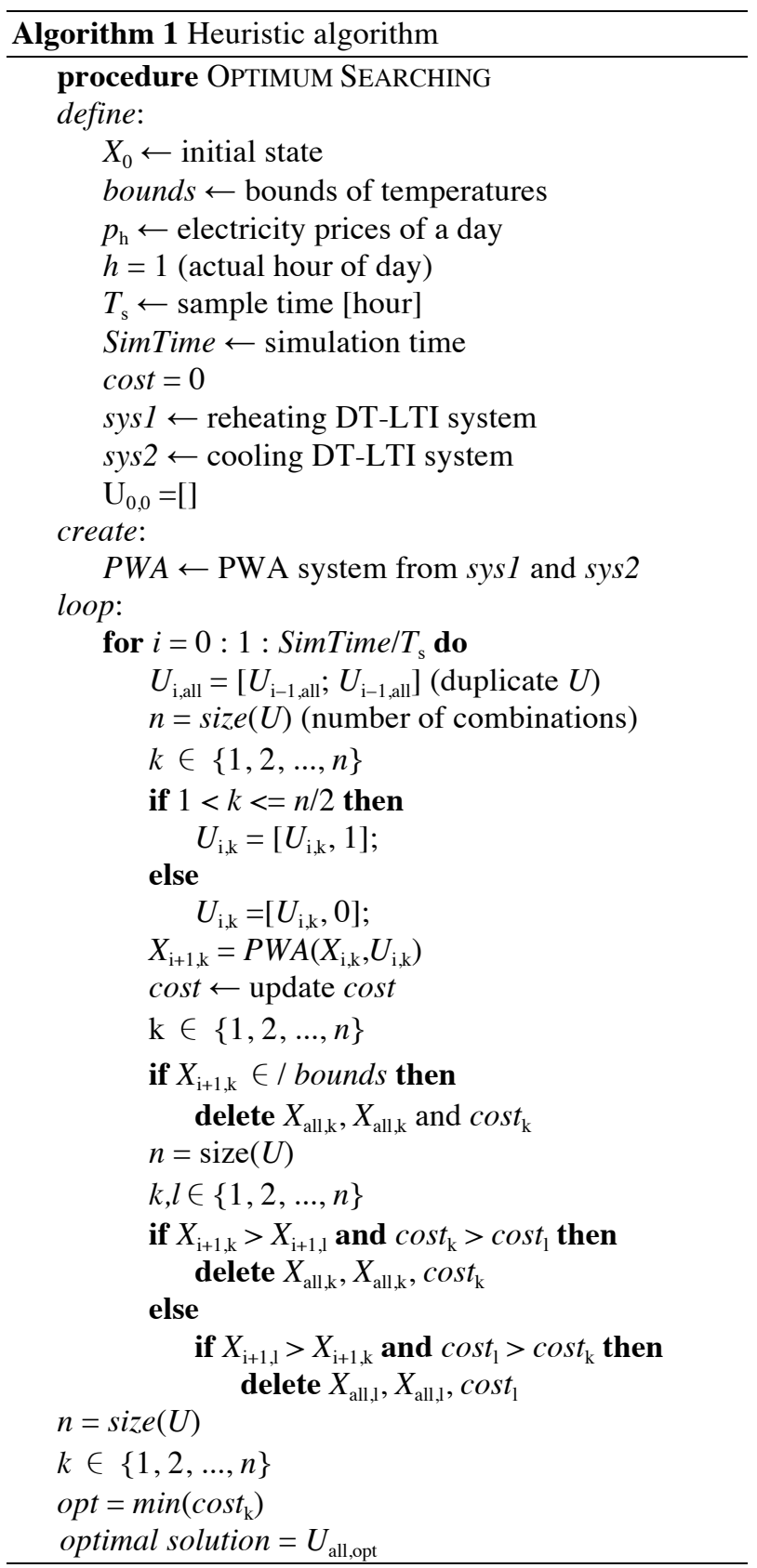

\subsubsection{Algorithm with Horizon}

The algorithm of the MPT uses a horizon to reduce the calculation time for fast online implementation. In the investigated problem, the maximum usable horizon available in MPT is too small. The previous algorithm calculates the optimal solution during a whole day, so the horizon is 24 hour, e.g. if the sample time is 5 minutes then the size of the horizon is 288 . This large horizon size causes slow simulations. To decrease this simulation time, a horizon as in the MPT algorithm is defined. This step causes a very large reduction in running time. This time is proportional to the horizon size, but if the horizon is too small the solution would not be optimal. The running times and calculated costs are collected in Table 2. 
The running times in Table 2 show that by increasing the horizon size the simulation time increases, too. The operating cost increases, but it is uncertain whether a bigger horizon is better. Based on this, the suggested heuristic search-based optimization algorithm can be used as a basis for a special model predictive scheduling/control algorithm.

\section{Results and Discussion}

The model predictive control algorithm in the MPT did not work correctly due to the serious limitations connected to the control task. By increasing the horizon size, the simulation becomes slow with maximum horizon size of 13 .

The proposed optimization algorithm has been developed to find the optimal scheduling. Table 2 shows the difference between simulation times and operating costs if the horizon size is changed. Fig.5 shows the calculated optimal scheduling for Wednesday. Fig.6 shows the energy prices and the number of input
Table 2. The size of horizon, the simulation time in minutes and the cost of best solution.

\begin{tabular}{crrr}
\hline $\begin{array}{c}\text { Size of } \\
\text { horizon }\end{array}$ & $\begin{array}{c}\text { Running } \\
\text { time }\end{array}$ & Cost & $\begin{array}{c}\text { Deviation } \\
\text { from optimal }\end{array}$ \\
\hline none $(\infty)$ & 75.0 & 0.20235 & $0 \%$ \\
24 & 5.7 & 0.20427 & $+0.95 \%$ \\
36 & 12.6 & 0.20433 & $+0.98 \%$ \\
48 & 16.9 & 0.20394 & $+0.79 \%$ \\
60 & 25.9 & 0.20413 & $+0.88 \%$ \\
72 & 31.0 & 0.20456 & $+1.09 \%$ \\
\hline
\end{tabular}

combinations of the $i^{\text {th }}$ cycle without and without a horizon size of 4 hours. There is a correlation between the monotonous energy price and the number of input combinations. If the price is increased the number opf combinations increases, too.

Simulations of energy prices for a whole week with and without horizon size of 4 hours $\left(T_{\mathrm{S}}=5\right.$ minutes) have also been performed. The results are shown in Table 3. The simulation times were reduced by $70-90 \%$ while the costs grew only by $1-2 \%$.
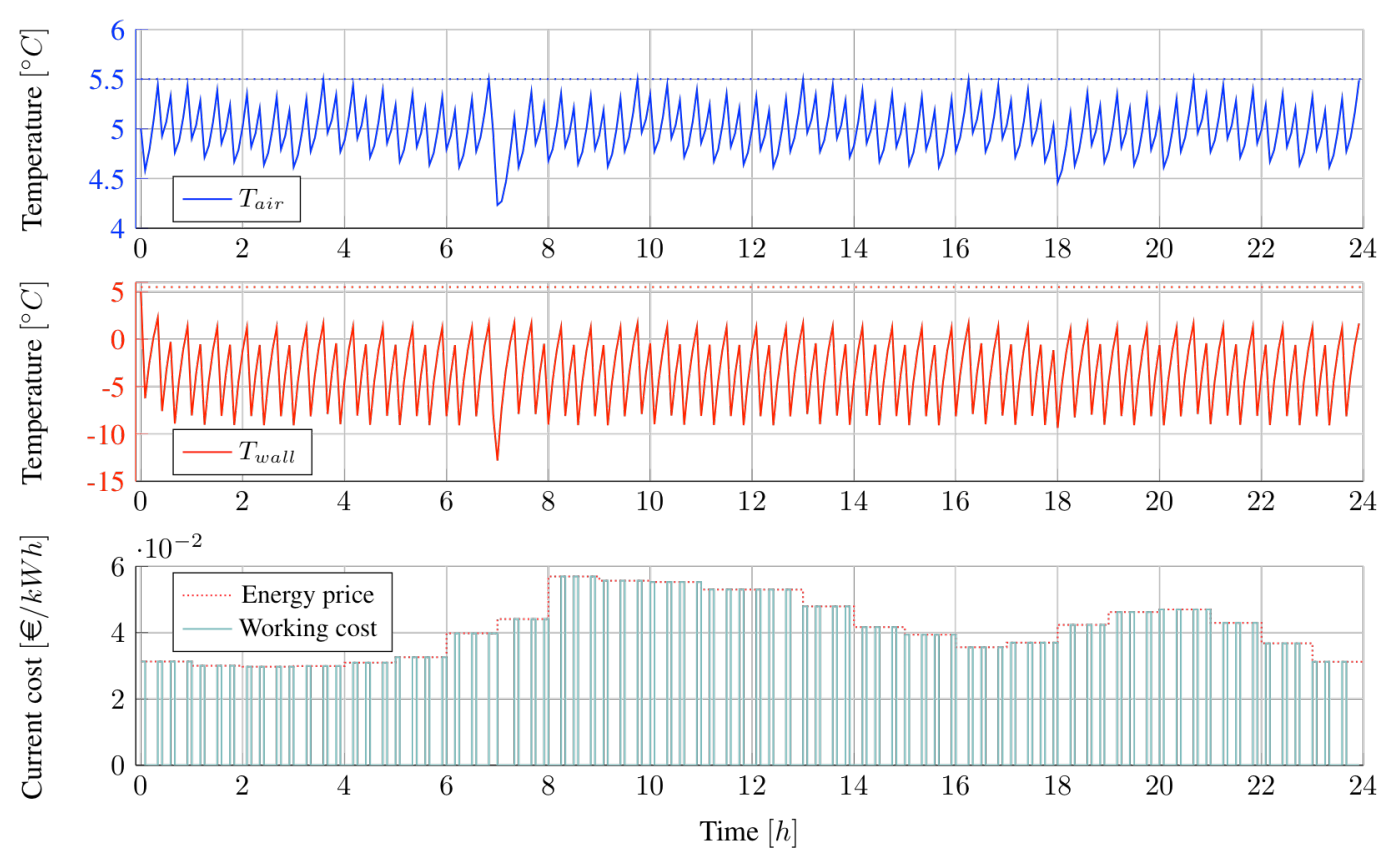

Figure 5. The optimal scheduling for Wednesday's energy prices.

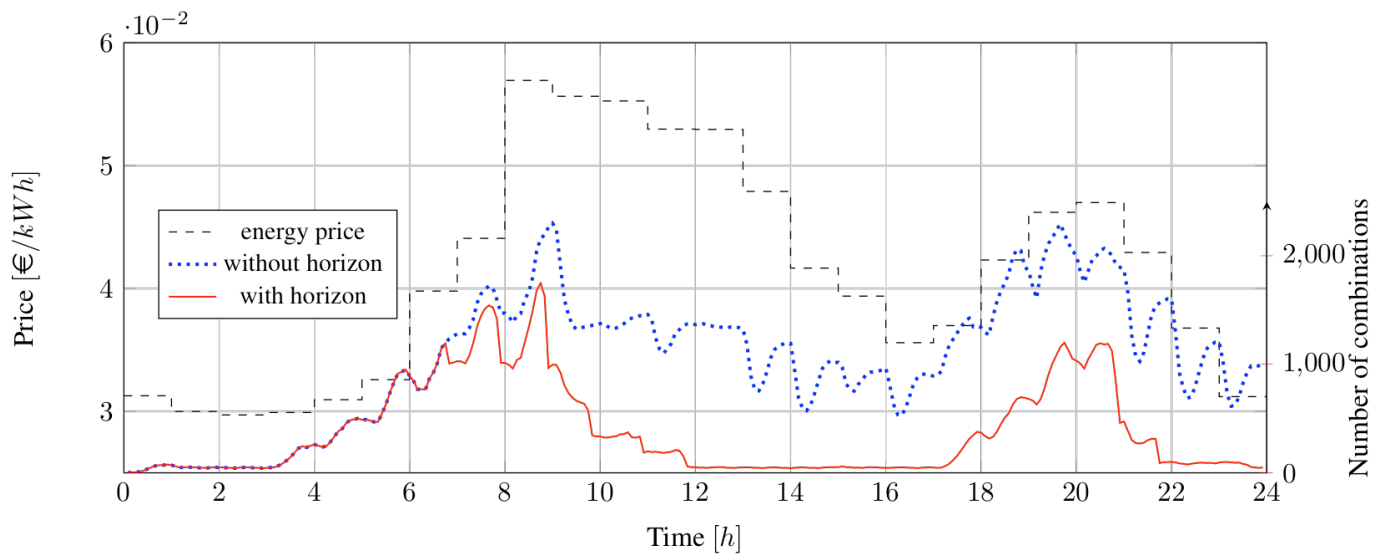

Figure 6. Wednesday energy prices and the number of input combinations of the $i^{\text {th }}$ cycle with and without a horizon size of 4 hours. 
Table 3. Differences in optimal operating cost and simulation time with and without a horizon.

\begin{tabular}{lrrrrrr}
\hline \multirow{2}{*}{ Day of the week } & \multicolumn{2}{c}{ without horizon } & \multicolumn{2}{c}{ 4 hour horizon } & \multicolumn{2}{c}{ difference in percent } \\
\cline { 2 - 7 } & cost, $1 / \mathrm{kW}$ & time, min & cost, $1 / \mathrm{kW}$ & time, min & cost, $1 / \mathrm{kW}$ & time, min \\
\hline Monday & 0.20235 & 75 & 0.20394 & 16.9 & +0.79 & -77.47 \\
Tuesday & 0.20200 & 1013.2 & 0.20650 & 78.9 & +2.23 & -92.21 \\
Wednesday & 0.27748 & 234 & 0.28111 & 48.8 & +1.31 & -79.18 \\
Thursday & 0.22255 & 116.1 & 0.22739 & 38.3 & +2.17 & -67.04 \\
Friday & 0.23127 & 267.7 & 0.23618 & 32.3 & +2.12 & -87.93 \\
Saturday & 0.25819 & 222.3 & 0.26281 & 45.1 & +1.79 & -79.70 \\
Sunday & 0.27286 & 100.4 & 0.27488 & 18.7 & +0.74 & -81.34
\end{tabular}

\section{Conclusion}

A heuristic search algorithm has been implemented for the optimal scheduling of a refrigerator model. This work was initiated by the fact that the optimization algorithm of the MPT did not work correctly with large horizon sizes for the applied piecewise affine system. The proposed algorithm calculates the optimal input sequence for a whole day with any horizon size but it also works with an infinitely large horizon. The calculation time is fast compared to that of the Matlab MPT solver.

Our own heuristic algorithm is faster without a horizon than the MPT solver with horizon size of 13 hours. I can be run with a larger horizon size, and can yield the absolute optimum without a prediction horizon. The operating cost of the solution is 12 percent less with the aid of a heuristic search. This cost is 25 percent cheaper than the cost of traditional refrigerator control.

The suggested heuristic search-based optimization algorithm can be used as a basis of a special model predictive scheduling/control algorithm. The present work is based on an ideal refrigerator model. As a future step, the validation of the model and the scheduling algorithm in an MPC framework will be investigated. Testing the control scheme in a real situation with random disturbances, e.g. opening the refrigerator's door, etc. will also be performed.

\section{SYMBOLS}

$\begin{array}{ll}\text { A } & \text { state matrix } \\ \mathbf{B} & \text { input matrix } \\ \mathbf{C} & \text { output matrix } \\ C_{\text {air }} & \text { heat capacity of the inside of the refrigerator } \\ C_{\text {wall }} & \text { heat capacity of the back-wall } \\ \text { DAM } & \text { Day-Ahead Market } \\ \text { DT-LTI } & \text { Discrete Time Linear Time Invariant } \\ \boldsymbol{f} & \text { constant input or disturbance } \\ \boldsymbol{\Phi}_{\text {off }} & \text { off state discrete time state matrix } \\ \boldsymbol{\Phi}_{\text {on }} & \text { on state discrete time state matrix } \\ \Gamma_{\text {off }} & \text { off state discrete time input matrix } \\ \Gamma_{\text {on }} & \text { on state discrete time input matrix } \\ \text { LTI } & \text { Linear Time Invariant } \\ \text { MPC } & \text { Model Predictive Control } \\ \text { MPT } & \text { Multi-Parametric Toolbox }\end{array}$

PWA Piecewise Affine

$\mathbf{Q}_{\mathrm{u}} \quad$ penalty matrix of $\boldsymbol{u}$ vector in MPT

$\mathbf{Q}_{\mathrm{x}} \quad$ penalty matrix of $\boldsymbol{x}$ vector in MPT

$R_{\text {cool }} \quad$ heat resistance of the back-wall

$R_{\mathrm{e}} \quad$ heat resistance between back-wall and inner air

$R_{\mathrm{i}} \quad$ insulation of refrigerator

$S \quad$ binary state switch

$T_{\text {air }} \quad$ inner air temperature of refrigerator

$T_{\text {out }} \quad$ outer room temperature

$T_{\text {wall }} \quad$ cooled back-wall temperature of refrigerator

$\boldsymbol{u}$ input vector

$\boldsymbol{u}_{\mathrm{k}} \quad$ input vector value in $k^{\text {th }}$ sample moment in DT

$U_{\text {air }} \quad$ voltage corresponding to inner air temperature

$U_{\text {cool }} \quad$ voltage corresponding to minimum reachable back-wall temperature

$U_{\text {out }} \quad$ voltage corresponding to outer air temperature

$U_{\text {wall }} \quad$ voltage corresponding to cooled back-wall temperature

$\boldsymbol{x} \quad$ state vector $\boldsymbol{x}_{\mathrm{k}} \quad$ state vector value in $k^{\text {th }}$ sample moment in
DT

\section{Acknowledgement}

This research is supported by the National Research, Development and Innovation Office - NKFIH through grant No. 115694. A. Magyar was supported by the János Bolyai Research Scholarship of the Hungarian Academy of Sciences. The authors would like to express their gratitude to Prof. Katalin Hangos and Dr. Gyula Simon for useful discussions.

\section{REFERENCES}

[1] Huisman, R.; Huurman, C.; Mahieu, R.: Hourly electricity prices in day-ahead markets, Energy Econ. 2007 29(2), 240-248 DOI 10.1016/j.eneco.2006.08.005

[2] Nord Pool: Elspot prices, www.nordpoolspot.com/ Market-data1/Elspot/Area-Prices/ALL1/Hourly, 2016

[3] Herceg, M.; Kvasnica, M.; Jones, C.; Morari, M.: Multi-parametric toolbox 3.0, Proc. Eur. Control Conf., EPFL-CONF-186265 (Zurich, Switzerland) 2013 